\title{
Calculation methods for plate and beam elements of box-type structure of building
}

\author{
Mirziyod Mirsaidov ${ }^{1 *}$, Makhamatali Usarov $^{2}$, and Giyosiddin Mamatisaev ${ }^{3}$ \\ ${ }^{1}$ Tashkent Institute of Irrigation and Agricultural Mechanization Engineers, Tashkent, Uzbekistan \\ ${ }^{2}$ Institute of Mechanics and Seismic Stability of Structures of the Academy of Sciences of the \\ Republic of Uzbekistan, Tashkent, Uzbekistan \\ ${ }^{3}$ Fergana Polytechnic Institute, Fergana, Uzbekistan
}

\begin{abstract}
The article is devoted to the dynamic calculation of the boxtype structure of buildings for seismic resistance, taking into account the spatial work of the box elements under the influence of dynamic impact. The development of spatial calculations and the study of vibrations of elements of box-type structures, considering various factors, is an urgent problem of structural mechanics. In this article, a mathematical model and a numerical-analytical method were developed to solve the problem of dynamics using the finite difference method and expand the solution in terms of natural vibration modes in the spatial setting for the elements of box-type structures under the kinematic impact. The steady-state forced vibrations of box-type structures under stationary harmonic influences applied to the structure foundation are investigated, and the areas are identified where the greatest values of shearing forces and bending moments occur under harmonic influences.
\end{abstract}

\section{Introduction}

Methods for calculating the strength of a structure and its elements are based on the theory of beams, plates and shells, which is one of the most important branches of mechanics. The achievements of the theory of bending, stability and vibrations of beams, plates and shells are widely used when solving the problem of seismic resistance of buildings and structures.

The study in [1] is devoted to static accounting for higher vibration modes in the problems of dynamics of building structures under external harmonic load. With a computational software package, the displacements of nodes and internal forces in the elements of the structures under consideration were determined.

The effect of displacements, fractures of the wall panel axes in the process of their installation on the operation of large-panel structures was considered in [2]. The analysis of design schemes was conducted with an account for different types of installation errors. Forces in structural elements exceeding the permissible ones were determined with an account for the error in the installation of parts.

* Corresponding authors:theormir@mail.ru 
During earthquakes, the degree of damage to buildings and structures depends on the characteristics of seismic impact (intensity, spectral composition, etc.). The reliable design and construction of the structures depend on the soil properties of the construction site foundation [3]-[7].

The studies in [8]-[10] are devoted to improving the box-type model of the building structure, taking into account the contact conditions between the elements of the panels and beams. Equations of motion of box-type elements and graphs of displacements of plates and beams are constructed. The article deals with the problem of forced vibrations of a building of spatial box type, which consists of rectangular panels and interacting beams under a dynamic action given by the foundation displacement according to the sinusoidal law. The method of finite differences was used to solve the problem.

The studies in[11], [12] develop the methods for dynamic spatial calculation of the structure based on the finite difference method in the framework of the theory of bimoments, taking into account the spatial stress-strain state.

[13], [14] are devoted to the numerical solution of the problem of transverse and longitudinal vibrations of buildings and structures based on a plate model developed within the framework of the bimoment theory of plates. The problems of transverse and longitudinal vibrations of buildings and structures are solved using the developed model within the bimoment theory of plates[15], [16].

The data on the mechanism of tsunami wave formation and destruction were analyzed in [17], the recommendations for tsunami-resistant construction were generalized. A solution was proposed to mitigate damage from strong earthquakes and high tsunami waves.

[18] discusses the main issues of determining the reinforcement parameters of reinforced concrete structures during their inspection. The basic ways to solve these problems are analyzed. The most reliable and accurate methods for determining the parameters of reinforcement are shown.

In [19]-[24], the dynamic characteristics and vibrations of various axisymmetric and plane structures are considered, taking into account various geometries, spatial factors and inelastic properties of materials. The solution to the problem is performed by the finite element method and by expanding the solution in terms of natural vibration modes. Various mechanical effects associated with the geometry of the structure and inelastic properties of the material are revealed.

For more precise stress and strain state definition for structures are vital put in calculation scheme the reliable constitutive relations. The physical reliability and new method for the solution of plasticity problems using Il'yushin's approximating relation are described here [25]. A model of plasticity for a transversely isotropic material with allowance for complex loading is developed based on the results of experiments[26]. Some plasticity problems regarding complex loading are obtained [27] also.

This is an overview of only several publications devoted to the methods for solving dynamic problems and the study of vibrations of the elements of box-type structures and other structures under various impacts. The above review shows the incompleteness of research in this direction; therefore, this article devoted to developing calculation methods and the study of vibrations of various elements of box-type structures presents a relevant problem of structural mechanics. 


\section{Methods}

a. Mathematical models of the problem

A spatial box consisting of beam and plate elements is taken as a design model of a building (Fig. 1). It is assumed that the bottom part of the box is rigidly fixed and, under dynamic action, moves together with the foundation.

The fixed bottom part of the box under seismic impact in the direction of the $\mathrm{OZ}$ axis moves according to a given harmonic law, i.e. $U_{0}(t)[8,9]$ :

$$
U_{0}=A_{0} \sin \omega_{0} t
$$

$A_{0}$ and $\omega_{0}$ are the amplitude and frequency of external influence.

Let us assume that the external load-bearing elements of the building (plate elements $1,3)$, located perpendicular to the direction of seismic impact, work only for transverse dynamic bending. Let us introduce designations for displacement induced by the strains in plate and beam elements. The deflections of the plate are indicated by $W(x, y, t)$. Side plate elements (plate elements 2,4), located in the direction of the external impact, are subjected to tension-compression and shear in their $O X Z$ plane.

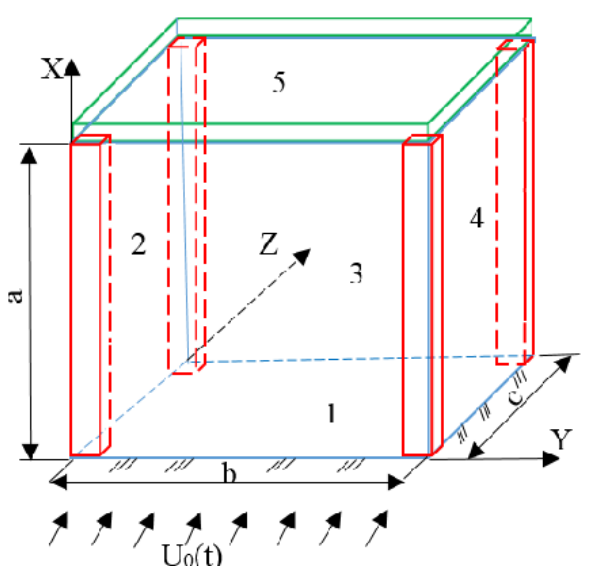

Fig. 1. Spatial box of a building

The displacements of the shear plate elements are indicated by $u(x, z, t), v(x, z, t)$. The plate elements of the box are connected by beam elements. Based on this condition, it follows that the beam elements are subjected to bending and torsion. Deflections and torsion angles of the beams are indicated by $W^{(i)}(x, t)$ and $\alpha^{(i)}(x, t)$, where: I, II, III, IV is the number of beams.

It is assumed that the floor slab (plate element 5) is also deformed. The law of motion of its points is determined following the deformation forms of the upper edges of the vertical contacting plate elements. The functions for floor slab points displacement are indicated by $u_{n}(z, y, t), v_{n}(z, y, t)$.

Further, the following designations are introduced for the plate elements of the building box: 
$E_{b}, \rho_{b}, h_{b}$ and $v_{b}$ are the elastic modulus, density, width, thickness and Poisson's ratio of bending plate elements $E_{c}, \rho_{c}, h_{c}$ and $v_{c}$ are the elastic modulus, density, width, thickness and Poisson's ratio of shear plate elements.

$E_{n}, \rho_{n}, h_{n}$ and $v_{n}$ are the modulus of elasticity, density, thickness and Poisson's ratio of the slab, $G_{n}$ is the shear modulus of the slab $G_{n}=\frac{E_{n}}{2\left(1+v_{n}\right)}$. The height of the box (the dimensions of plate and beam elements are the same) is $h=a$. It is considered that all beam elements have a square cross-section $h_{b}, h_{b}$ made of the same material. Modules of elasticity and shear of beams are indicated by $E$ and $G$, Poisson's ratio - by $v$, density - by $\rho$, moments of inertia of the beam section under bending and torsion - by $J$ and $I_{k r}$.

The following internal force factors are introduced, i.e. the forces and moments arising in the box elements and in the zones of their connection. The expressions for the bending and torque moments $M_{x x}, M_{y y}$ and $M_{x y}$ of the bent plate elements are introduced by the following formulas

$$
\begin{aligned}
& M_{x x}=-D\left(\frac{\partial^{2} W}{\partial x^{2}}+v_{b} \frac{\partial^{2} W}{\partial y^{2}}\right), M_{y y}=-D\left(v_{b} \frac{\partial^{2} W}{\partial x^{2}}+\frac{\partial^{2} W}{\partial y^{2}}\right), \\
& M_{x y}=-D\left(1-v_{b}\right) \frac{\partial^{2} W}{\partial x \partial y}
\end{aligned}
$$

where: $D=\frac{E_{b} h_{b}^{3}}{12\left(1-v_{b}^{2}\right)}$ is the cylindrical stiffness under transverse bending.

The expressions for the longitudinal and tangential forces of plate shear elements are given in the following form:

$$
P_{z z}=B\left(\frac{\partial u}{\partial z}+v_{c} \frac{\partial v}{\partial x}\right), P_{x x}=B\left(v_{c} \frac{\partial u}{\partial z}+\frac{\partial v}{\partial x}\right), P_{x z}=\frac{B\left(1-v_{c}\right)}{2}\left(\frac{\partial u}{\partial x}+\frac{\partial v}{\partial z}\right)
$$

where: $B=\frac{E_{c} h_{c}}{1-v_{c}^{2}} 0$ is the cylindrical stiffness of plate elements under tension and compression.

Bending and torsional moments of beams are:

$$
M^{(i)}=-E J \frac{\partial^{2} W^{(i)}}{\partial x^{2}}, M_{k r}^{(i)}=E I_{k r} \frac{\partial \alpha^{(i)}}{\partial x}
$$

Where $E I_{k r}$ is the torsional rigidity of the beam, $E J$ is the bending stiffness of the beam. 
The expression for the shear force of the plates bent in the zones of their connections with the beams is as follows:

$$
\begin{aligned}
& R_{x}=Q_{x}+\frac{\partial M_{x y}}{\partial x}, R_{y}=Q_{y}+\frac{\partial M_{x y}}{\partial y}, \\
& Q_{x}=-D \frac{\partial}{\partial x}(\Delta W) \\
& Q_{y}=-D \frac{\partial}{\partial y}(\Delta W) .
\end{aligned}
$$

Longitudinal and tangential forces of plate elements in contact zones, working in shear, have the following expressions:

$$
P_{z}^{c}=B\left(\frac{\partial u}{\partial z}+v_{c} \frac{\partial v}{\partial x}\right)_{z=c_{i}}, \quad P_{z x}^{c}=\frac{B\left(1-v_{c}\right)}{2}\left(\frac{\partial u}{\partial x}+\frac{\partial v}{\partial z}\right)_{z=c_{i}} .
$$

Where $b i$ and $c i$ are the corresponding coordinates of beam-and-column elements.

The displacement field of the beam-and-column elements is determined based on the Bernoulli hypothesis in the form

$$
v=-z \frac{\partial W}{\partial x}
$$

The torsion of the beam-and-column element is induced by the bending moments at the edges of the plate elements 1 or 3 .

b. Method for solving the problem.

An analytical-numerical method is proposed for solving the problem of box-type structure vibrations, taking into account spatial strains with complete contact conditions in the zones of joints of plate and beam elements of the structure box.

Based on representation (1), we rewrite the kinematic laws of displacement of points of plate elements. The general kinematic law of motion of the box is presented as the sum of the function of the foundation displacement $U_{0}(t)$ and the relative displacements of plates and beams

$$
\begin{aligned}
& u_{3}=U_{0}(t)+W(x, y, t) \\
& u_{1}=U_{0}(t)+u(x, z, t), u_{2}=v(x, z, t) \\
& u_{3}^{(i)}=U_{0}(t)+W^{(i)}(x, y, t)
\end{aligned}
$$

The displacements of plate and beam elements are given in the following form: 


$$
\begin{aligned}
& W=W(x, y) \sin \left(\omega_{0} t\right), \\
& u=u(x, z) \sin \left(\omega_{0} t\right), v=v(x, z) \sin \left(\omega_{0} t\right), \\
& W^{(i)}=W^{(i)}(x, y) \sin \left(\omega_{0} t\right), \alpha^{(i)}=\alpha^{(i)}(x, y) \sin \left(\omega_{0} t\right) .
\end{aligned}
$$

Consider a theoretical calculation of the building box under the dynamic impact, taking into account the spatial work of transverse and longitudinal plate elements.

Let us compose the equations of motion for each plate and beam elements of the boxtype structure of a building [8-10]. Plate elements are considered to be thin elastic plates obeying the Kirchhoff-Love hypothesis. Each beam is subject to bending and torsion. When constructing the equation of motion and the boundary conditions of the plates and beams, the expressions for the moments of forces and displacements (2) - (6) are used.

The system of equations for bending, torsional moments and shear forces is presented in the following form

$$
\begin{aligned}
& \frac{\partial M_{x x}}{\partial x}+\frac{\partial M_{x y}}{\partial y}-Q_{x}=0, \\
& \frac{\partial M_{x y}}{\partial x}+\frac{\partial M_{y y}}{\partial y}-Q_{y}=0, \\
& \frac{\partial Q_{x}}{\partial x}+\frac{\partial Q_{y}}{\partial y}=\rho_{b} h_{b} \ddot{W}+\rho_{b} h_{b} \ddot{U}_{0} .
\end{aligned}
$$

The system of equations of motion of shear plate elements is taken as:

$$
\begin{aligned}
& \frac{\partial P_{z z}}{\partial z}+\frac{\partial P_{x z}}{\partial x}=\rho_{c} h_{c} \ddot{u}+\rho_{c} h_{c} \ddot{U}_{0}, \\
& \frac{\partial P_{z x}}{\partial z}+\frac{\partial P_{x x}}{\partial x}=\rho_{c} h_{c} \ddot{v} .
\end{aligned}
$$

The system of equations for bending and torsional vibrations of the beams is:

$$
\begin{aligned}
& \frac{\partial M^{(i)}}{\partial x}-Q^{(i)}+\frac{h_{b}}{2} P_{z x}^{c}=0, \\
& \frac{\partial Q^{(i)}}{\partial x}+\rho_{\mathrm{i}} F \ddot{W}^{(i)}=R_{y}-P_{z}^{c}-\rho_{\mathrm{i}} F \ddot{U}_{0}, \\
& \frac{\partial M_{k r}^{(i)}}{\partial x}=\rho_{i} I_{\kappa r} \ddot{\alpha}^{(i)}+M_{y y}^{b}+\frac{h_{c}}{2} R_{y} .
\end{aligned}
$$

where: $G I_{k r}$ is the torsional rigidity of the beam.

The boundary conditions at the foundation of the building box are written as for rigid fixing. The lower part of the building moves with the foundation, and there is no torsion. 


$$
u_{1}=u_{3}=u_{3}^{(i)}=U_{0}(t), u_{2}=0, \frac{\partial W}{\partial x}=0, \frac{\partial W^{(i)}}{\partial x} 0, \alpha^{(i)}=0
$$

Boundary conditions (12) with (7) are rewritten as:

$$
W=0, \frac{\partial W}{\partial x}=0, u=0, v=0, W^{(i)}=0, \frac{\partial W^{(i)}}{\partial x}=0, \alpha^{(i)}=0
$$

The contact conditions in the zone of connection of beam and plate elements working in shear are written in the following form

$$
\begin{aligned}
& u(x, z, t)_{z=c_{i}}=W^{(i)}(x, t), \quad v(x, z, t)_{z=c_{i}}= \pm \frac{h_{b}}{2} \frac{\partial W^{(i)}(x, t)}{\partial x} \\
& W(x, y, t)_{y=b_{i}}=W^{(i)}(x, t),\left(\frac{\partial W(x, y, t)}{\partial y}\right)_{y=b_{i}}=-\alpha^{(i)}
\end{aligned}
$$

The displacements of the upper points of the beam and plate elements working in bending and shear are denoted by

$$
W_{a}(y, t)=W(a, y, t), u_{a}(y, t)=u(a, z, t), v_{a}(z, t)=v(a, z, t) .
$$

Based on the notation (15), the distribution law for the displacements of the floor slab points are given by the following expressions

$$
\begin{aligned}
& u_{n}(z, y, t)=W_{a}(y, t)+u_{a}(z, t)-W^{(i)}(a, t) \\
& v_{n}(z, y, t)=v_{a}(z, t) .
\end{aligned}
$$

The boundary conditions at the upper ends of the elements of the building box at $x=a$ are the following contact conditions between these elements and the floor.

The contact conditions at the joints of the floor and plate bending elements have the following form

$$
-R_{x}+\eta_{0} \rho_{n} h_{b} h_{n} \ddot{W}_{a}=h_{b} h_{n} \frac{\partial \tau_{z y}^{n}}{\partial y}-\eta_{0} \rho_{n} h_{b} h_{n} \ddot{U}_{0}, M_{x x}^{a}=0
$$


where: $\tau_{z y}^{n}=G_{n}\left(\frac{\partial W_{a}}{\partial y}\right)$ is the shear stress of the floor at its edge $z=c$, $\eta_{0}=\frac{2\left(b h_{b}+c h_{c}\right)}{b c}$.

Contact conditions at the joints of the floor and beam-column elements are written as:

$$
\begin{aligned}
& M^{(i)}=0, \quad-Q^{(i)}+\eta_{0} \rho_{n} h_{b} h_{n} \ddot{W}^{(i)}=-\eta_{0} \rho_{n} h_{b} h_{n} \ddot{U}_{0}, \\
& M_{k r}^{(i)}=0 .
\end{aligned}
$$

The contact conditions at the joints of the floor and plate shear elements, relative to the contact tangential and normal stresses, are written in the following form:

$$
\begin{aligned}
& -c h_{c} \tau_{z x}^{c}+\eta_{0} m_{n c} \ddot{u}_{a}=c h_{c} h_{n} \frac{\partial \sigma_{z z}^{n}}{\partial z}-\eta_{0} m_{n c} \dot{U}_{0} \\
& -c h_{c} \sigma_{x x}^{c}+\eta_{0} m_{n c} \ddot{\cup}_{a}=c h_{c} h_{n} \frac{\partial \sigma_{x z}^{n}}{\partial z}
\end{aligned}
$$

where: $\sigma_{z z}^{n}=E_{n}\left[\frac{\partial u_{a}}{\partial z}\right], \sigma_{z x}^{n}=G_{n}\left[\frac{\partial v_{a}}{\partial z}\right]$ are the normal and shear stresses of the floor at its edge $y=b, \sigma_{x x}^{c}=E_{c}\left[\frac{\partial v}{\partial x}+v_{c} \frac{\partial u}{\partial z}\right]_{x=a}, \tau_{z x}^{c}=G_{c}\left[\frac{\partial v}{\partial z}+\frac{\partial u}{\partial x}\right]_{x=a}$ are the normal and shear stresses of shear plate elements at its upper edge $x=a$.

The boundary conditions on the contour of the window opening are set as for the free edge (Fig. 2):

$$
\begin{gathered}
M_{x x}=0, R_{x}=0, \text { at } x=\text { const },(\text { on the contour of } \mathrm{AB} \text { and } \mathrm{CD}), \\
M_{y y}=0, R_{y}=0, \text { at } y=\text { const },(\text { on the contour of } \mathrm{AC} \text { and } \mathrm{BD}) .
\end{gathered}
$$

At each interior corner points of the opening A, B, C, D, we have five boundary conditions:

$$
M_{x x}=0, R_{x}=0, M_{y y}=0, R_{y}=0, M_{x y}=0, \text { at } x=\text { const, } y=\text { const }
$$




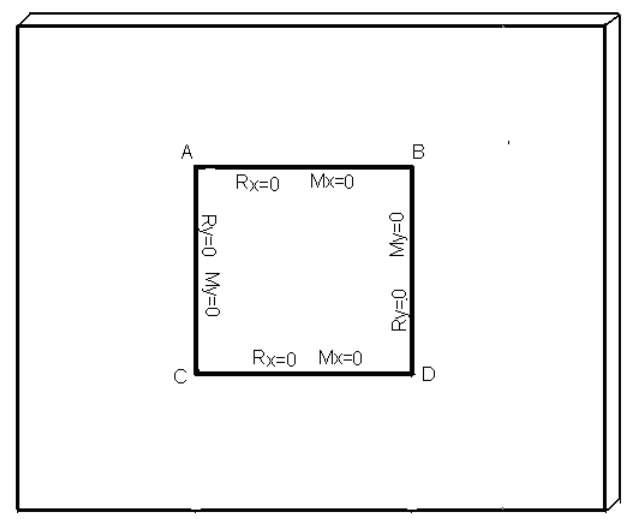

Fig. 2. Bending plate element with cutout

The vibration modes (8) must satisfy the equations of motion (9) - (11), boundary conditions (13), (19), and (20), and contact conditions (14), (17), and (18).

The general solution to the problem of forced vibrations of bending plate elements of the box is described by a function represented as the sum of the solution to the problem of forced and natural vibrations:

$$
W(x, y, t)=A_{0} W_{v}(x, y) \sin \omega_{0} t+\sum_{i=1}^{N} C_{i} W_{i}(x, y) \sin p_{i} t
$$

The solution to the problem of forced vibrations of a plate element in bending is expressed through natural vibration modes[19-21]

$$
W_{v}(x, y)=\sum_{i=1}^{N} A_{i} W_{i}(x, y)
$$

where $A_{i}(i=1,2,3, .$.$) are the expansion coefficients.$

In the calculations, it was sufficient to restrict ourselves to the one-term approximation. The general solution to the equation of bending vibrations of the panels is taken in the following form:

$$
W(x, y, t)=A_{0} W_{v}(x, y) \sin \omega_{0} t+C_{1} W_{1}(x, y) \sin p_{1} t,
$$

Let us express the solution to the problem of forced vibrations in terms of the natural vibration mode

$$
W_{v}(x, y)=A_{1} W_{1}(x, y),
$$

where: $p_{1}$ is the first eigen frequency, $W_{v}(x, y)$ is the mode of forced vibrations, $C_{1}$ is a constant to be determined. 
Substituting (24) into (23) and using zero initial conditions, we obtain $C_{1}=-A_{0} A_{1} \frac{\omega_{0}}{p_{1}}$. By virtue of this expression and taking into account (24), we obtain the general solution of the problem for bending plate elements in the following form [8-10]:

$$
W(x, y, t)=A_{0}\left(\sin \omega_{0} t-\frac{\omega_{0}}{p_{1}} \sin p_{1} t\right) W_{v}(x, y) .
$$

The expression for displacement of shear plate elements has the form

$$
\begin{aligned}
& u(x, z, t)=A_{0}\left(\sin \omega_{0} t-\frac{\omega_{0}}{p_{1}} \sin p_{1} t\right) u_{v}(x, z), \\
& u(x, z, t)=A_{0}\left(\sin \omega_{0} t-\frac{\omega_{0}}{p_{1}} \sin p_{1} t\right) v_{v}(x, z) .
\end{aligned}
$$

The kinematic functions of the beams are written as:

$$
\begin{aligned}
& W^{(i)}(x, t)=A_{0}\left(\sin \omega_{0} t-\frac{\omega_{0}}{p_{1}} \sin p_{1} t\right) W_{v}^{(i)}(x), \\
& \alpha^{(i)}(x, t)=A_{0}\left(\sin \omega_{0} t-\frac{\omega_{0}}{p_{1}} \sin p_{1} t\right) \alpha_{v}^{(i)}(x) .
\end{aligned}
$$

The problem of determining the unknown coordinate functions in expressions (25) (27) is solved by the finite difference method.

For this purpose, in this study, a method (based on the method of finite difference schemes) was developed for the dynamic calculation of the box-type structure of buildings.

\section{Results and Discussion}

In the calculations, the value of the frequency of external influence is $\omega_{0}=81.03 \mathrm{sec}^{-1}$ , and the amplitude of the foundation displacement is $A_{0}=2 \mathrm{sm}$.

The following parameters are set as the initial data for the box elements.

Elastic moduli of plate elements in bending and shear are: $E_{b}=20000 \mathrm{MPa}$, $E_{c}=7500 \mathrm{MPa}$. Poisson's ratio of the materials of plate elements is $v_{b}=v_{c}=v_{c}=0.3$.

The geometrical dimensions are given as: 
for bending plate elements 1 and 3 the thickness is $h_{b}=0.5 \mathrm{~m}$, the height is $a=3,25 \mathrm{~m}$, and the length is $b=4 \mathrm{~m}$, and for plate elements 2 and 4 the thickness is $h_{c}=0,25 \mathrm{~m}$; the length is $c=6 \mathrm{~m}$.

Let us proceed to a discussion of the numerical results obtained for the bending moment on bent plate elements.

Figures 3 and 4 show the graphs of the time variation of the deflections at the upper points of the plate elements working in bending, without considering the initial conditions (dashed line) and with the initial conditions (solid line).

As can be seen, the deflection values increase up to $20 \%$ (Fig. 3.) when considering the initial conditions of the problem.

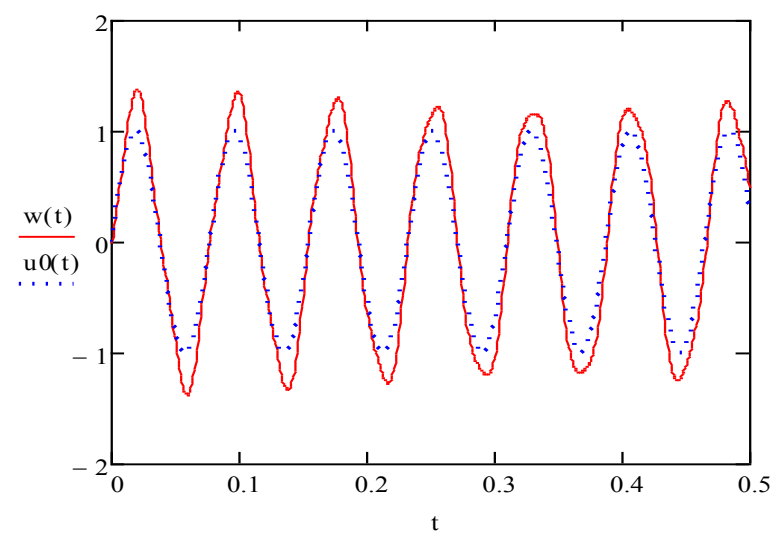

Fig 3. Change of deflections in time at the upper points (at $x=a, y=b / 2$ ) of plate bending elements

Figure 4 shows a graph of the change in bending moment $M_{y y}$ on the upper and lower parts of the bent plate elements. Analysis of the results obtained (Fig. 4) shows that the values of bending moment $M_{y y}$ in the middle of the panel are less than at the edges of the plate elements. 


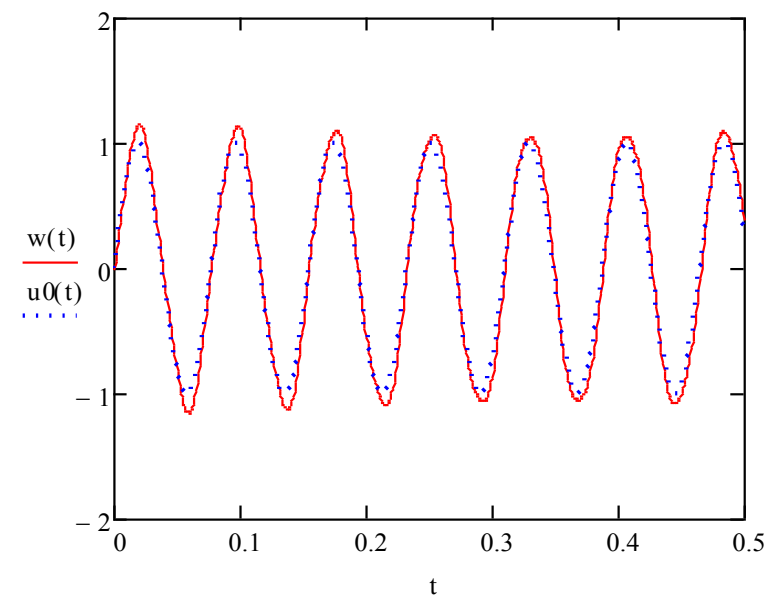

Fig 4. Change in deflections in time at the upper points (at the midpoint at $x=a / 2, y=b / 2$ ) of plate bending elements

The maximum value of bending moment $M_{y y}$ was determined in the middle of the second half of the contact zone of bent plate and beam elements. Note that on the contact zones of the plate elements, the bending moment $M_{y y}$ twists the beams and plays the role of the torque.

a)

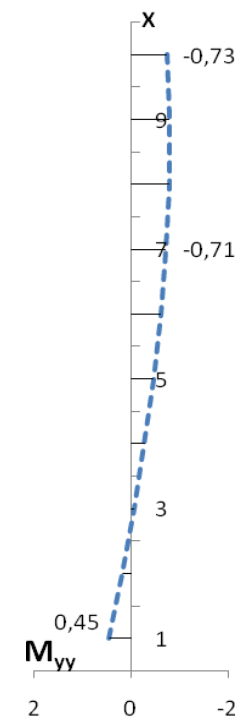

b)

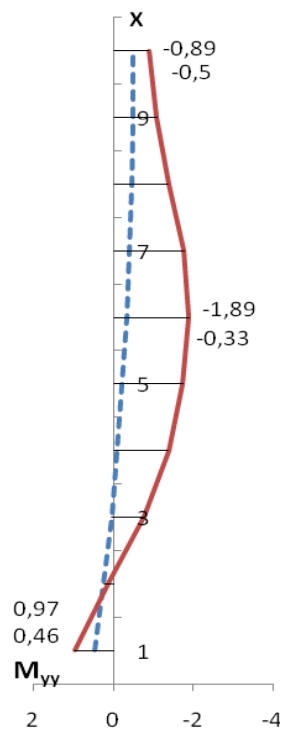

c)

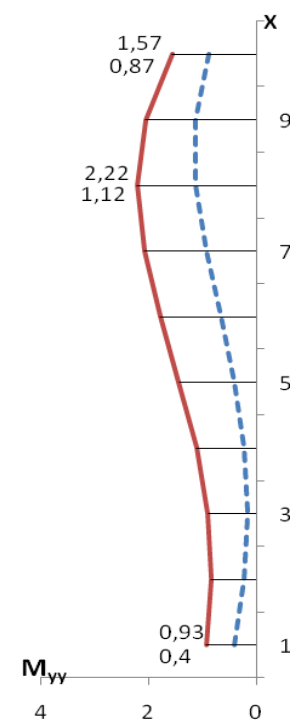

Fig. 4. Change in bending moments $M_{y y}$ in plate bending elements in height: a) at the edge of the window opening b) at the middle part c) at the right edge of plate elements 
Further, Figures 5 and 6 show the values of the moments and shear forces obtained when solving stationary problems without considering the window openings (dashed lines) and with the window openings(solid lines).

Figure 6 shows a graph of the change in shear forces $Q_{y y}$ in bent plate elements. Analysis of these results shows that the values of shear forces $Q_{y y}$ at the edge of the plate elements are greater than at the middle part.

a)

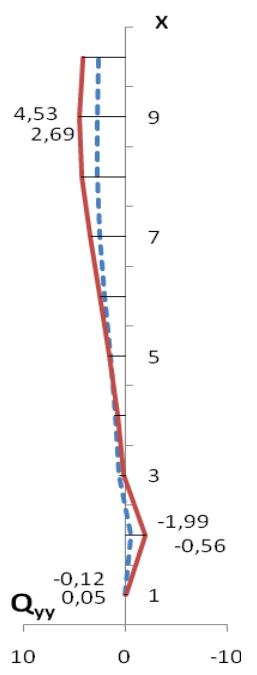

b)

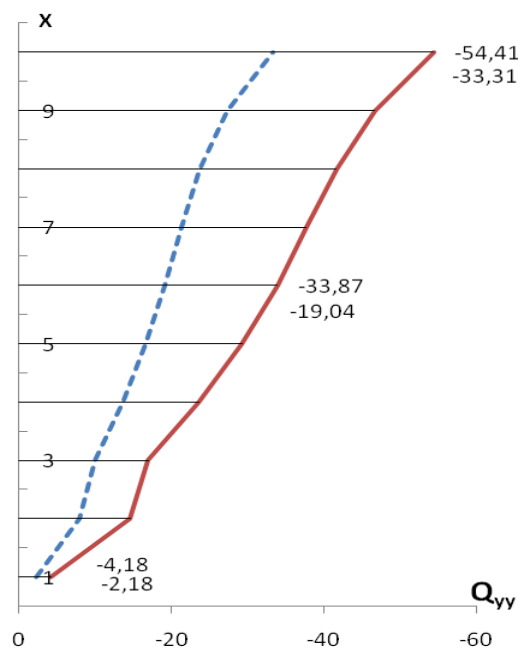

Fig. 6. Change in height of shear forces $Q_{y y}$ in plate bending elements: a) at the middle part b) at the right edge of the plate elements

Analysis of the results (Fig. 5) showed that the maximum value of shear force $Q_{y y}$ is observed in the upper part of the plate and beam elements connection zone.

\section{Conclusions}

1. A mathematical model was developed for dynamic calculations of plate and beam elements of box-type structures of buildings, taking into account the spatial nature of their work under various kinematic impacts.

2. A numerical-analytical method was developed to study the forced vibrations of elements of box-type structures of a building using the finite difference method and the expansion of the solution in terms of natural spatial modes of vibrations.

3. The steady-state forced vibrations of box-type structures under stationary harmonic kinematic effect on the foundation were investigated. 
4. Investigations of the internal force factors arising in the elements of the box-type structure have shown the areas of the box-type structure, where the greatest values of shear forces and bending moments occur under harmonic influences.

\section{References}

1. Le, T.Q.T., Lalin, V. V., Bratashov, A.A. Static accounting of highest modes in problems of structural dynamics. Magazine of Civil Engineering. 2019. DOI:10.18720/MCE.88.1.

2. Vatin, N.I., Kuznetsov, V.D., Nedviga, E.S. Installation errors in calculating largepanel buildings. Magazine of civil engineering. 2011. DOI:10.5862/mce.24.3.

3. Pshenichkina, V.A., et al. Methodology for assessing the seismic reliability of buildings with high floors. Bulletin of the Volgograd State University of Architecture and Civil Engineering. Series: Construction and Architecture. 25. pp.50-56, (2011)

4. Khachatryan, S.O. Spectral-wave theory of earthquake resistance. Earthquake engineering. Safety of facilities. 3. pp.58-61, (2004)

5. Radin, V.P., Trifonov, O.V., Chirkov, V.P. A model of a multi-story frame building for calculations of intense seismic effects. Earthquake-resistant construction. Safety of facilities. 1. pp.23-26, (2001)

6. Tyapin, A.G. Calculation of structures for seismic effects, taking into account interaction with a soil base. M.: DIA Publishing House, p.399, (2013)

7. Chopra, A.K. Elastic response spectrum: a historical note. Earthquake Engineering and Structural Dynamics. 36(1). pp. 3-12, (2007)

8. Usarov, M., Mamatisaev, G. Calculation on seismic resistance of box-type structures of large-panel buildings. IOP Conf. Series: Materials Science and Engineering. (2020). 971. Pp. 032041. DOI:doi:10.1088/1757-899X/971/3/032041.

9. Usarov, M., et al. Building oscillations based on a plate model. IOP Conference Series: Materials Science and Engineering. (2020). DOI:10.1088/1757$899 x / 883 / 1 / 012211$.

10. Usarov,M et al. Dynamic calculation of boxed design of buildings. IOP Conf. Series: Materials Science and Engineering. 2020. 883(012186.). DOI:10.1088/1757-899X/883/1/012186.

11. Yarashov, J., Usarov, M., Ayubov, G. Study of longitudinal oscillations of a fivestorey building on the basis of plate continuum model. E3S Web of Conferences. (2019). DOI:10.1051/e3sconf/20199704065.

12. Toshmatov, E., et al. Dynamic methods of spatial calculation of structures based on a plate model. E3S Web of Conferences. 2019. DOI:10.1051/e3sconf/20199704072.

13. Usarov, D., Turajonov, K., Khamidov, S. Simulation of free vibrations of a thick plate without simplifying hypotheses. Journal of Physics: Conference Series. (2020). DOI:10.1088/1742-6596/1425/1/012115.

14. Usarov, M.K. Buckling of orthotropic plates with bimoments. Magazine of Civil Engineering. 2015. DOI:10.5862/MCE.53.8.

15. Usarov, M., et al. To the theory of bending and oscillations of three-layered plates with a compressible filler. IOP Conference Series: Materials Science and Engineering. (2020). DOI:10.1088/1757-899X/869/5/052037.

16. Abdikarimov, et al. Free oscillations of three-layered plates. IOP Conference Series: Materials Science and Engineering. (2020). DOI:10.1088/1757899x/883/1/012058.

17. Belash, T.A., Yakovlev, A.D. Seismic stability of a tsunami-resistant residential 
buildings. Magazine of Civil Engineering. (2018). DOI:10.18720/MCE.80.9.

18. Ulybin, A.V. Inspection methods of reinforcement parameters of concrete structures. Magazine of Civil Engineering. (2012). 27(1). Pp. 4-13. DOI:DOI: 10.5862/MCE.27.

19. Mirsaidov, M., Mekhmonov, Y. Nonaxisymmetric vibrations of axisymmetric structures with associated masses and hollows (protrusions). Strength of Materials. March. 1987. 19(3). Pp. 424-430. DOI:10.1007/BF01524147.

20. Ishmatov, A.N., Mirsaidov, M. Nonlinear vibrations of an axisymmetric body acted upon by pulse loads. Soviet Applied Mechanics. 1991. DOI:10.1007/BF00896519.

21. Mirsaidov, M., Troyanovskii, I.E. Forced axisymmetric oscillations of a viscoelastic cylindrical shell. Polymer Mechanics. (1975). DOI:10.1007/BF00857626.

22. Sultanov K.S., Vatin, N.I. Wave Theory of Seismic Resistance of Underground

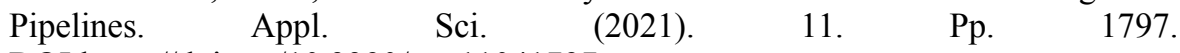
DOI:https://doi.org/10.3390/app11041797.

23. Sultanov, K.S., et al. Quasistaticity of the process of dynamic strain of soils. Magazine of Civil Engineering. 2019. DOI:10.18720/MCE.85.7.

24. Normuminov, B., et al. Parametric vibrations of viscoelastic orthotropic cylindrical panels of variable thickness. IOP Conference Series: Materials Science and Engineering. (2020). DOI:10.1088/1757-899X/869/5/052034.

25. Babamuratov, K.S., Abirov, R.. On Physical Reliability in the Theory of Plasticity. Strength of Materials. (2001). 33. Pp. DOI:DOI:org/10.1023/A:1010478208161.

26. Abirov, R.A. Allowance for complex loading in transversely isotropic solids. Journal of Applied Mechanics and Technical Physics. (2009). DOI:10.1007/s10808-009-0015-0.

27. Abirov, R.A. On the physical reliability and taking complex loading into account in plasticity. Materials Science. (2008). DOI:10.1007/s11003-009-9114-6. 